This process may evidently be continued. We may then state the following

Theorem: The $r$ th polar of $B$ with respect to $C_{n}$ is $C_{n-r}$.

II.

Again let there be three distinct points $A, B$, and $C$ on the same straight line $l$, and through the point $C$ let the line $l_{1}$ be drawn perpendicular to $l$. Let lines $l_{2}$ and $l_{3}$ be drawn through $A$ and $B$ respectively, and let $l_{2}$ and $l_{3}$ intersect on $l_{1}$. Let $l_{2}$ make an angle $\alpha$ with $l$, and $l_{3}$ make an angle $\beta$ with $l$, and let a line $l_{4}$ be drawn through $B$, making an angle $n \beta$ with $l$. Let $l_{2}$ and $l_{4}$ intersect in $D$. Then just as in section I, the equation representing the locus of $D$ is

$$
\begin{aligned}
k\left[x^{n}-\left(\begin{array}{c}
n \\
2
\end{array}\right) x^{n-2} y^{2}+\cdots\right] & \\
= & (x-c)\left[\left(\begin{array}{c}
n \\
1
\end{array}\right) x^{n-1}-\left(\begin{array}{c}
n \\
3
\end{array}\right) x^{n-3} y^{2}+\cdots\right],
\end{aligned}
$$

where $k=(a-c) / a$ and $a=A C$, and $c=A B$.

It is then evident that the theorem in section I holds for the curve represented by equation(7).

Ohio State University.

\title{
ON THE RECTIFIABILITY OF A TWISTED CUBIC.
}

BY DR. MARY F. CURTIS

(Read before the American Mathematical Society, April 27, 1918.)

GIven the twisted cubic

$$
x_{1}=a t, \quad x_{2}=b t^{2}, \quad x_{3}=c t^{3}, \quad a b c \neq 0 ;
$$

to show that the condition that it is a helix is precisely the condition that it is algebraically rectifiable.

If (1) is a helix, then $T / R$, the ratio of curvature to torsion, is constant. Denoting differentiation with respect to $t$ by 
primes, we have

$$
\begin{aligned}
& x^{\prime}: \quad a \quad 2 b t \quad 3 c t^{2}, \\
& x^{\prime \prime}: \quad 0 \quad 2 b \quad 6 c t \text {, } \\
& x^{\prime \prime \prime}: 006 \text {, } \\
& \left(x^{\prime} \mid x^{\prime}\right)=a^{2}+4 b^{2} t^{2}+9 c^{2} t^{4}, \quad\left(x^{\prime \prime} \mid x^{\prime \prime}\right)=4\left(b^{2}+9 c^{2} t^{2}\right), \\
& \left(x^{\prime} \mid x^{\prime \prime}\right)=2 t\left(2 b^{2}+9 c^{2} t^{2}\right), \quad\left|x^{\prime} x^{\prime \prime} x^{\prime \prime \prime}\right|=12 a b c, \\
& \left(x^{\prime} x^{\prime \prime} \mid x^{\prime} x^{\prime \prime}\right)=\left(x^{\prime} \mid x^{\prime}\right)\left(x^{\prime \prime} \mid x^{\prime \prime}\right)-\left(x^{\prime} \mid x^{\prime \prime}\right)^{2} \\
& =4\left(a^{2} b^{2}+9 a^{2} c^{2} t^{2}+9 b^{2} c^{2} t^{4}\right) \text {. } \\
& \frac{T}{R}=-\left(\frac{x^{\prime} x^{\prime \prime} \mid x^{\prime} x^{\prime \prime}}{x^{\prime} \mid x^{\prime}}\right)^{3 / 2} \frac{1}{\left|x^{\prime} x^{\prime \prime} x^{\prime \prime \prime}\right|} \text {. }
\end{aligned}
$$

Since $\left|x^{\prime} x^{\prime \prime} x^{\prime \prime \prime}\right|$ is constant, $T / R$ is constant when and only when $\left(x^{\prime} x^{\prime \prime} \mid x^{\prime} x^{\prime \prime}\right) /\left(x^{\prime} \mid x^{\prime}\right)$ is constant. We thus have

$$
4\left(a^{2} b^{2}+9 a^{2} c^{2} t^{2}+9 b^{2} c^{2} t^{4}\right) \equiv \rho\left(a^{2}+4 b^{2} t^{2}+9 c^{2} t^{4}\right) ;
$$

hence $\rho=4 b^{2}$ and $9 a^{2} c^{2}-4 b^{4}=0$. Conversely, for all values of $a, b, c, a b c \neq 0$, for which

$$
9 a^{2} c^{2}-4 b^{4}=0,
$$

$T / R$ is constant-in particular, is equal to $\mp 1$, according as $2 b^{2}= \pm 3 a c$ - and the cubic ( 1 ) is a helix.

If we had fixed our attention on another characteristic property of a helix, namely, that the tangent makes with a fixed direction a constant angle, we should have again derived the condition (2). The fixed direction-that of the axis of the cylinder on which the helix lies-is $(1 / \sqrt{2}, 0, \pm 1 / \sqrt{2})$ and the helix cuts the rulings of the cylinder under an angle of $45^{\circ}$.

That (2) is a necessary and sufficient condition that $s$, the arc of (1), is an algebraic function of $t$ and hence that (1) is algebraically rectifiable follows from the fact that the integral

$$
s=\int_{t_{0}}^{t} \sqrt{a^{2}+4 b^{2} t^{2}+9 c^{2} t^{4}} d t
$$

is algebraic when and only when (2) holds. Hence the theorem: The twisted cubic (1) is algebraically rectifiable when and only when it is a helix. 